Aus der Königlichen chirurgischen Universitätsklinik zu Göttingen (Direktor: Geh. Med.-Rat Prof. Dr. H. B r a u n).

\title{
Über die isolierte Ausreißung der Ligamenta cruciata des Kniegelenks.
}

\author{
Von Dr. Otto Köhler, \\ Oberarzt im Ulanenregiment Hennigs von Treffenfeld (Altmärkischen) Nr. I6, \\ kommandiert zur Klinik.
}

(Mit 3 Abbildungen.)

Es ist allgemein bekannt, daß ein Teil unserer Gelenkbänder so kräftig entwickelt ist, daß, wenn sie bei gewaltsamer Gelenkbewegung äußerst stark angespannt werden, nicht etwa zerreißen, sondern intakt bleiben und einen Knochenbruch herbeiführen. Als charakteristische Beispiele brauche ich hier nur den Knöchelbruch und den Bruch der unteren Radiusepiphyse anzuführen. Von den Gelenkbändern des Kniegelenks wissen wir, daß die Seitenbänder bei schwerer Distorsion des Gelenks dünne Rindenstücke aus ihrem Ansatze reißen können und daß durch das Ligamentum patellare gelegentlich ein Abriß der Tuberositas tibiae herbeigeführt wird.

Eine ganz hervorragende Festigkeit haben die beiden Ligamenta cruciata des Kniegelenks. Dies kann uns auch nicht wundern, wenn wir uns daran erinneren, welche schweren Anforderungen ihre Funktionen, nämlich die Erhaltung der Gelenkflächen in gegenseitiger Berührung und Hemmung der Streckbewegung, an ihre Stärke stellen. Die Festigkeit dieser glatten, massigen Gebilde ist so groß, daß sie, wie die eingehenden Versuche an

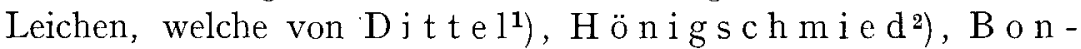

I) D it te l, Leopold, Über intraartikuläre Verletzungen am Knie. Wiener med. Jahrbücher 1876 , S. 3 I 9 .

2) $\mathrm{Hönigschmied,} \mathrm{Johann,} \mathrm{Leichenexperimente} \mathrm{über} \mathrm{die} \mathrm{Zer-}$ reißungen der Bänder im Kniegelenk. Deutsche Zeitschr. f. Chir. 1893, Bd. 36, S. 587 . 
Über die isolierte Ausreißung der Ligamenta cruciata des Kniegelenks. 22 I

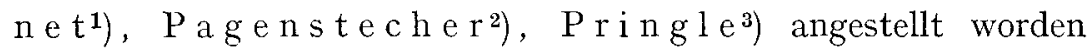
sind und auf die ich später noch zurückkommen werde, sowie die wenigen klinischen Beobachtungen lehren, bei Bewegungen, die über die funktionellen Grenzen hinausgehen, in ihrer Mitte, oder doch so, daß ein längerer Stumpf übrigbleibt, nie zerreißen. Sie lösen sich vielmehr (ganz oder teilweise) von ihren Knochenansätzen ab oder sie reißen so dicht an denselben ab, daß nur ein Stümpfchen noch haften bleibt. Als Beweis für die Unzerreißbarkeit der Ligamenta cruciata in der Mitte führe ich einen Autor an, der sich direkt über diesen Punkt ausspricht. D i t t e 1 (l. c. S. 33I) sagt: ,Was ich aus den Experimenten und der einzigen klinischen Beobachtung über die intraartikuläre Verletzung am Knie lernen konnte, läßt sich in folgendes zusammendrängen: I. Die Kreuzbänder zerreißen nie, d. h. in i h r e r M i t t e, sie können aber durch die oben angeführte gewaltsame, über die funktionellen Grenzen hinausgehende Bewegung abgelöst werden, und zwar lösen sie sich fast konstant von ihrem Femoralende ab; durch Überstreckung das hintere, durch Überbeugung das vordere." Hiermit befindet sich in Übereinstimmung das Ergebnis einer Arbeit aus der neuesten Literatur von $\mathrm{F}$ e B le $\mathrm{r}^{4}$ ), der die Dehnbarkeit, Elastizitätsbreite und Zugfestigkeit der Bänder einer genaueren Prüfung unterzogen hat. Er stellte fest, daß die Bänder in der Mitte fester sind als an ihrem Ansatz, so daB sie zuerst an diesem abreißen.

Beim Studium der einschlägigen Literatur ist es mir nur einmal gelungen, eine Angabe über Zerreißung der Kreuzbänder in ihrer Mitte zu finden. H ö $\mathrm{n}$ i g s ch m i e d sagt (1. c. S. 6r 7 ): „Bei den Leichenversuchen, wo die Rotation nach außen mit großer Gewalt ausgeführt wurde, war das Ligamentum cruciatum anterius einmal vom Femur losgetrennt, und dreimal war es i n d e r M i t t e

I) Bonnet, Über die Krankheiten der Gelenke. Übersetzt von Krupp. I 847 , S. 488 .

2) P a g e n t e cher, Die isolierte Zerreißung der Kreuzbänder des Knies. Deutsche med. Wochenschr. 1903, Nr. 47, S. 872.

3) Pringle, Avulsion of the spine of the tibia. Annals of surgery 1907 August. Referat im Zentralbl. f. Chir. I907, Bd. 34, Nr. 49, S. I430.

4) Zitiert nach R. Fick, Handbuch der Anatomie und Mechanik der Gelenke I904, Teil I, S. 30 . 
zerrissen." Meines Erachtens handelt es sich hier um Ausnahmefälle, deren Zustandekommen schwer erklärbar und wohl von Faktoren abhängig ist, die ich später noch besprechen werde. Dann findet sich bei $\mathrm{K}$ ö $\mathrm{n} \mathrm{i} \mathrm{g}^{\mathbf{1}}$ ) (S. 68) eine Angabe, die ich hier erwähnen möchte. Bei der Besprechung der Zerreißungen der Kreuzbänder sagt er, daß sie auch in der Kontinuität zerreißen können. Man kann nicht umhin, hierunter eine Zerreißung in der Mitte oder ungefähr in der Mitte zu verstehen, besonders da $\mathrm{K}$ ö n i g neben dieser Zerreißung ,,in der Kontinuität" die ,am Knochen" anführt. Ich habe nicht feststellen können, worauf der Autor seine Angabe stützt, es geht auch aus seinen drei eigenen Beobachtungen nicht hervor.

Im Anschlusse hieran möchte ich darauf hinweisen, daß die Bezeichnungsweise der Kreuzbandverletzungen in der Literatur nicht immer ganz exakt ist. Durchweg wird jede Verletzung der Kreuzbänder als „Zerreißung“ bezeichnet, wo die Bezeichnung „Abreißung“ oder ,Ablösung“ richtiger wäre. Öfters kommt es auch vor, daß ein Autor ein- und dieselbe Verletzung in dem einen Satz als ,Zerreißung", in dem nächsten als „Abreißung“" usf. bezeichnet. Meines Erachtens wäre es besser, wenn scharf zwischen ,Zerreißung“, ,Abreißung oder Ablösung“" und ,Ausreißung mit Knochenstück" unterschieden und die erste Bezeichnung nur für solche Fälle gebraucht würde, bei denen die Verletzung in der Mitte oder doch ungefähr in der Mitte erfolgte.

Nicht selten kommt nun vor, daß ein Kreuzband beim Abreißen ein Knochenstück von seiner Ansatzstelle mitnimmt. Für diesen Fall würde die soeben erwähnte Bezeichnung ,Ausreißung mit Knochenstück" gut passen. Am Knochen ist dann immer ein der Größe des ausgerissenen Stückes entsprechender Defekt vorhanden. Die Ansatzstelle kann ganz oder teilweise ausgerissen werden; öfters werden auch nur mehrere kleinere Knochenstückchen ausgerissen, die dann am Bande hängen. Daß derartige Knochenstücke eine recht ansehnliche Größe haben können, wird uns der von $\mathrm{D}$ it t e l beobachtetc klinische Fall, in welchem das Knochenstück eine Länge von $3 \mathrm{~cm}$ und eine Breite von $4 \mathrm{~cm}$

I) K ö n i g, Fritz, Beiträge zur Gelenkchirurgie. Archiv f, klin. Chir. I906, Bd. 8I, Teil II, S. 65. 
hatte, und ein in der hiesigen Klinik zur Begutachtung gekommener Fall zeigen. Ausgerissen wird sowohl die Einpflanzungsstelle am Femur als die an der Tibia. Wie aus den Leichenexperimenten hervorgeht, ist der Sitz des Ausrisses am Femur das Gewöhnliche. Der Sitz des Ausrisses an der Tibia ist dagegen sehr selten. B arth $\mathbf{1}$ ), der, worauf ich schon jetzt hinweisen möchte, auf die Ausreißung von Knochenstücken durch die Bänder als wichtigste Entstehungsursache für die traumatischen Gelenkkörper nachdrücklich hinweist, sagt (1. c. S. 566) am Schlusse seiner Statistik, daß die Kondylen des Femurs die häufigste Ursprungsstätte der traumatischen Gelenkkörper des Knicgelenks sind. Von 30 seiner 55 Fälle, über die bestimmte Angaben gemacht sind, entfallen 24, d. i. 8 o Proz., auf die Kondylen des Femurs, 4, d. i. 3 Proz., auf die Patella und nur 2, d. i. 6,6 Proz., auf die Gelenkfläche der Tibia.

Als letzte Verletzung, die ein Kreuzband erleiden kann, hätte ich danı noch eine Beschädigung zu nennen, für die die Bezejchnung ,Längszerreißung“ gecignet wäre. Dieselbe ist zweifellos sehr selten. Ich habe sie in der Literatur nur einmal beschrieben gefunden, und zwar bei dem zweiten von Pagenstecher (1. c.) boobachteten Fall. Der Autor fand nach Eröffnung des Gelenks, da $B$ das Ligamentum cruciatum anterius in der hinteren Partie seines Ansatzes am Condylus lat. fem. durchtrennt, aber nicht ausgerissen war, so daß noch ein kleiner Stumpf am Condylus haftete; von der Rißstelle erstreckte sich ein Iängsriß bis fast zur tibialen Insertion.

Die soeben besprochenen Kreuzbandverletzungen - in nachstchender Arbeit möchte ich mich mit der einen Gruppe von ihnen näher beschäftigen, mit den Knochenausreißungen - gewinnen nun ein besonderes Interesse, wenn sie nur an ein e m Kreuzbande zustande kommen, ohne daß das andere Kreuzband und der übrige Bandapparat die geringste Beschädigung zeigen. Die ,,isolierte Kreuzbandzerreißung" galt bislang als ungemein selten. In der älteren und neueren Literatur findet man, wie dies auch von $\mathrm{K} \ddot{o} \mathrm{n}$ i g festgestcllt worden ist, nur wenige Beobachtungen. Wie außerordentlich selten und einzig dastehend diese Verletzung

I) B a r th, Arthur, Die Entstehung und das Wachstum der freien Gelenkkörper. Archiv f. klin. Chirurg. 1898, Bd. 56, S. 507 . 
von D ittel (l. c.) aufgefaßt wurde, geht am deutlichsten aus der Schilderung hervor, die er von der ,,höchst sonderbaren“ Verletzung in seinem bereits erwähnten Fall gibt. Nachdem der Autor die Bedingungen, unter denen die Verletzung zustande gekommen war, festgestellt hatte, wartete er ,mit Spannung“ die Sektion des inzwischen septisch gewordenen Patienten ab, um die Verletzung am anderen Kniegelenk experimentell $\mathrm{zu}$ erzeugen. ,Längere Zeit wollte das Experiment nicht gelingen, die ersten Hiebe hatten keinen Erfolg, endlich vernahm ich das ersehnte Krachen, und es wurde möglich, eine größere Verschiebbarkeit am Gelenke zu erzeugen, und so beendigte ich das Experiment, welches ich in Gegenwart des Herrn Prof. Hof mann und meiner Herren Sekundärärzte anstellte. Als ich das Gelenk eröffnete und untersuchte, war meine Befriedigung eine vollständige, denn ich hatte dieselbe intraartikuläre Verletzung erzeugt, nämlich Abhebung einer Knochenscheibe mit der Eminentia intercondyloidea bei unverletztem Bandapparate. Eine Verletzung, welche hier, solange ein Rokitansky gewirkt hat, ihm nicht vorgekommen war. Seitdem wiederholte ich öfters das Experiment mit gleichem Erfolge." Auch aus der im Jahre I903 veröffentlichten Abhandlung von Pagenstecher (l. c.), der drei wertvolle selbst beobachtete Fälle von isolierter Abreißung eines Kreuzbandes bringt und über Leichenversuche, die er an diese Beobachtungen anschloß, berichtet, geht hervor, daß er die ,isolierte Zerreißung" der Kreuzbänder für höchst selten hält. L e s e r ${ }^{\mathbf{1}}$ ) äußert sich folgendermaßen über diesen Punkt: ,Was die Bänderzerreißungen des Kniegelenkes angeht, so sind dieselben als isolierte Verletzungen nicht sehr häufig“", und W i $1 \mathrm{~m} \mathrm{~s}^{2}$ ) sagt sogar ganz allgemein, daß wir über die Zerreißungen der im Kniegelenk verlaufenden Bänder nicht viel wissen. Es war zuerst $\mathrm{K}$ ö n i g (1. c.), welcher, bestärkt durch drei eigene Beobachtungen, darauf aufmerksam machte, daß die Seltenheit der isolierten Kreuzbandzerreißung überschätzt würde. Er weist darauf hin, daß die Kreuzbandverletzungen oft das der Gelenkmaus eigentümliche Krank-

I) L e s e r, Edmund, Die spezielle Chirurgie in 60 Vorlesungen I 908 , 8. Aufl., S. IIOI.

2) Wullstein u. Wil ms, Lehrbuch der Chirurgie, II. Teil, I909, S. 287 . 
heitsbild haben und sich deshalb in den hierher gehörenden Abhandlungen finden. In der Tat findet man $z$. B. in der von $B$ a $\mathrm{t} \mathrm{h}$ (l. c. S. 557) aufgestellten Statistik eine Reihe von Fällen von Gelenkkörpern, deren Entstehung nur durch isolierte Kreuzbandausreißung erklärt werden kann. Ferner betont $\mathrm{K}$ ö $\mathrm{nig}$ die Wichtigkeit der vor wenigen Jahren noch unbekannten Röntgenographie, die uns heutzutage ein unentbehrliches Hilfsmittel bei der Diagnose der Ausreißung von Knochenstücken geworden ist. Im ersten seiner Fälle konnte er 1/4 Jahr nach der Verletzung mit Hilfe des Röntgenbildes die Diagnose auf Knochenabriß stellen; die stereoskopische Aufnahme zeigte recht deutlich den Sitz des Stückes vorn auf der Tibia, welcher Befund durch die Operation bestätigt wurde. Und im zweiten Falle konnte er durch das Röntgenbild einen kleineren Knochenausriß an der Eminentia intercondylica der Tibia nachweisen. Hieran anschließend möchte ich bemerken, daß ein gutes, deutliches Bild von besonderer Wichtigkeit für die Diagnose ist; ferner daß mehrere Autnahmen gemacht werden und daß auf allen derselbe Knochenschatten wieder hervortritt, wenn es sich um kleinere Knochenstücke handelt. Ein Kontrollbild des gesunden Knies halte ich für unerläßlich. Eine stereoskopische Aufnahme ist wünschenswert, weil sie uns den genaueren Sitz des Knochenstücks anzeigen kann. $Z$ weifellos wird auf diese Weise künftig mancher verdächtige Fall klargestellt werden, der vor der Röntgenära einfach unerkannt blieb.

Man kann sich wohl der $\mathrm{K}$ ö n ig schen Ansicht, daß die Seltenheit des Vorkommens der isolierten Kreuzbandzerreißung etwas überschätzt wird, anschließen. Ich für meine Person tue es auf Grund des Studiums der einschlägigen Literatur und weil ich selbst über drei Beobachtungen von Ausreißung von Knochenstücken durch das Ligamentum cruciatum anterius verfüge. Da dieselben mancherlei Interessantes bieten, möchte ich sie im Nachstehenden mitteilen. Das eine Mal erfolgte die Ausreißung aus der Insertion an der Innenfläche des äußeren Condylus des Femurs, die beiden anderen Male aus der Insertion in der Grube vor der Eminentia intercondylica tibiae.

Die erste Beobachtung betrifft einen Unfallpatienten, der von Professor $\mathrm{Br}$ a $\mathrm{u} \mathrm{n}$ untersucht und begutachtet wurde. 
Es handelte sich um einen Maurergesellen, der am 20. V. I903 einen Unfall dadurch erlitt, daß ihm beim Abputzen einer Stallwand ein Rind, welches vorgeführt werden sollte, gegen die Innenseite des rechten Kniegelenks sprang und ihm dasselbe nach außen umbog, so daß er hinfiel. Sein Arzt stellte Quetschung der Innenseite des rechten Kniegelenks, Schmerzhaftigkeit, Schwellung und Blaufärbung durch Bluterguß fest.

Patient wurde nun von Zeit zu Zeit von seinem Arzte begutachtet Die jedesmaligen Untersuchungsergebnisse waren nach den Akten kurz folgende.

I4. VIII. I903. Der Verletzte, der täglich schon wieder mehrere Kilometer gehen kann, klagt nur bei Überanstrengung über Schmerzen im rechten Kniegelenk sowie über Schwächegefühl im ganzen Bein. Rechtes Kniegelenk um $3 \mathrm{~cm}$ stärker als das linke; Umfang über die Mitte der Kniescheibe gemessen rechts 38 , links $35 \mathrm{~cm}$, oberhalb der Kniescheibe war das rechte Bein $2 \mathrm{~cm}$ stärker als das linke. Anschwellung an der Innenseite und oberhalb der Kniescheibe. Schleimbeutel neben und oberhalb der Kniescheibe mit flüssigem verschieblichen Inhalt. Beweglichkeit aktiv und passiv ziemlich frei, Streckung nicht ganz ausführbar.

8. X. I903. Angeblich jetzt Schmerzen an der Innenseite des rechten Kniegelenks, besonders nach längerem Gehen. Umfang über die Mitte der Kniescheibe rechts $34,5 \mathrm{~cm}$, links $32,5 \mathrm{~cm}$. Die Beweglichkeit war schon sehr gut, jedoch die Knochen noch verdickt und die Bänder noch zu schwach.

2. XI. 1903. Begutachter gibt an, daß jetzt infolge der Erschlaffung des Bandapparates des verletzten Knies eine Umbiegung des Kniegelenks nach außen eingetreten sei.

6. XII. I903. Beweglichkeit im rechten Kniegelenk gut, wenig eingeschränkt, besonders bei Beugung. Nirgends Anschwellung. Rechtes Kniegelenk bei Betrachtung und Betastung dicker wie das linke. Beim Strecken an der rechten Seite der Kniescheibe eine tiefe Furche. Rechts außen Verdickung des Knochenfortsatzes des Unterschenkels und Verknöcherung des Bandapparates. Bei Bewegungen zeitweise Reibegeräusche hörbar. Umfang über die Mitte der Kniescheibe $34 \mathrm{~cm}$.

6. III. Ig04. Bein noch schwach und schmerzhaft. Umfang über die Mitte der Kniescheibe $35 \mathrm{~cm}$. Beim Aufrechtstehen starkes Hervortreten des Gelenkfortsatzes des Unterschenkels rechts und außen von der Kniescheibe. Durchdrücken des Knies nicht ganz möglich. Zwischen Kniescheibe und dem nach außen gelegenen Knochenvorsprung eine tiefe Furche sicht- und fühlbar. Unterhalb der Kniescheibe eine druckempfindliche, plastische Schwellung. Gelenk beim Gehen gut beweglich, etwas nach außen stehend. Beim Betasten an der Innenseite der Kniescheibe reibende und knarrende Körper fühlbar.

23. IV. I904. Ergebnis der Untersuchung in der Klinik. 
Klagen über starke Schmerzen im rechten Kniegelenk beim Gehen, so $\mathrm{daß}$ nur leichte Arbeit möglich ist, und über Schwäche im linken Bein. Großer, kräftiger Mann von 43 Jahren. Rechts genu varum. Abmagerung des rechten Kniegelenks, so daß die Knochen desselben, besonders die Knorren des Oberschenkelbeins deutlicher fühlbar sind wie links. Bewegungen des Gelenks fast in normalen Grenzen möglich, nur Durchdrücken nicht ganz möglich. Bei Bewegungen manchmal, nicht immer, leichtes Knirschen fühlbar. Längenunterschied des rechten Ober- und Unterschenkels gegenüber links nicht vorhanden. Größter Umfang des rechten Oberschenkels $52 \mathrm{~cm}$ (gegen $52 \mathrm{~cm}$ links), Io $\mathrm{cm}$ oberhalb der Kniescheibe $40,2 \mathrm{~cm}$ (gegen $40,8 \mathrm{~cm}$ links), dicht oberhalb der Kniescheibe $34 \mathrm{~cm}$ (gegen $34,5 \mathrm{~cm}$ links), um die Mitte der Kniescheibe $35,5 \mathrm{~cm}$ (gegen $35 \mathrm{~cm}$ links); größter Umfang der rechten Wade $34,8 \mathrm{~cm}$ (gegen $34,2 \mathrm{~cm}$ links). Im Röntgenbild (siehe Fig. I) innerhalb des Kniegelenks in der Fossa intercondyloidea femoris, den untersten Teil derselben ausfüllend, ein längliches Knochenstück. An der Innenseite des Condylus lateralis femoris ein anfangs dreieckiger, dann sich verschmälernder, schräg nach oben und außen verlaufender Spalt sichtbar. Die den Spalt von oben her begrenzende Knochenmasse hängt mit dem erwähnten Knochenstück zusammen. Das Röntgenbild des linken Kniegelenks zeigt regelrechte Verhältnisse.

Auf Grund dieses Untersuchungsergebnisses und der Vorgeschichte gab Geheimrat B ra un sein Gutachten für

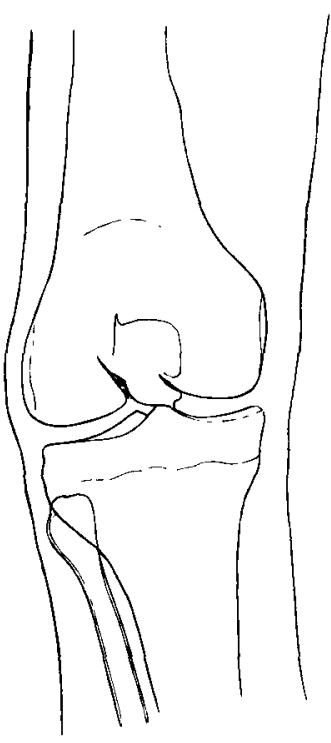

Fig. I. die Berufsgenossenschaft dahin ab, daß der Mann bei seinem Unfall einen im Inneren des rechten Kniegelenks gelegenen Knochenbruch erlitten habe, und zwar sei durch $Z$ ug des vorderen Kreuzbandes infolge des Umbiegens des rechten Knies nach außen ein Stück Knachen aus dem Femur ausgerissen worden. Da das Knochenstück nicht im mindesten die Erscheinungen einer Gelenkmaus bot, wurde angenommen, daß es wieder fest angehejlt war. Auch die fehlerhafte Stellung des rechten Beins in der Kniegelenksgegend wurde als Folge der Ausreißung aufgefaßt.

Die zweite und dritte Beobachtung machte ich im letzten Winter zu verschiedenen Zeitpunkten bei den Präparierübungen 
auf der hiesigen Anatomie. Es handelte sich um zwei Leichen, deren rechte Kniegelenke äußerlich nicht die geringste Spur einer Verletzung gezeigt hatten. Bei der Präparation fand sich nun bei beiden eine teilweise Ausreißung des Ligamentum cruciatum anterius aus seiner Insertion an der Tibia, während sonst an den Gelenken, bei denen bei der Präparation ganz besonders auf den Bandapparat geachtet wurde, keine Regelwidrigkeit festgestellt werden konnte. Ein Gelenkkörper fand sich in beiden Kniegelenken nicht. Da der Befund mich interessierte, wurden mir die Gelenke von dem Direktor des Anatomischen Instituts Herrn Geheimrat M e rkel freundlichst überlassen.

Das erste Präparat betrifft das rechte Kniegelenk eines Erwachsenen (Fig. 2). Der vordere Teil, etwa zwei Drittel des Lig. cruc. ant., welches sonst von regelrechter Beschaffenheit ist, ist aus seiner Insertion vor der Eminentia intercondyloidea tibiae herausgerissen. Die herausgerissenen Faserbündel ragen einzeln frei in das geöffnete Kniegelenk. An ihren Enden hängt je ein Knochenstückchen von der Größe eines Stecknadelkopfes bis zu der eines Kirschkerns. Letztere haben eine im ganzen rundliche Gestalt. Ihr Aussehen ist wie abgeschliffen; man sieht an ihnen keine Zacken, wie dies sonst an herausgerissenen Knochenstüçken der Fall ist. Besonders interessant ist das am meisten in das Gelenk hineinragende, etwa erbsengroße Knochenstuick. Dieses hängt an einem $3 / 4 \mathrm{~cm}$ langen Stiel von Fasern, die genau die obere Hälfte der Halbkugel umfassen, während die untere Hälfte entblößten Knochen zeigt. Der Stiel, der sich nach oben zu verschmälert und an seiner Abgangsstelle am Kreuzbande ganz dünn ist, ist um seine Längsachse gedreht. Hebt man den Stiel in die Höhe, so sieht man vereinzelte kurze Bandfasern, an denen ganz kleine, kaum stecknadelkopfgroße, rundliche Knochenstückchen hängen. An der Stelle der Tibia, an der sich die Insertion des Kreuzbandes befunden hatte, erblickt man eine zweipfennigstückgroße Grube, deren Grund höckrig und mit einer grauen Gewebsschicht bedeckt ist. An der lateralen Seite der Grube befindet sich ein etwa kirschkerngroßes Knochenstück, das hinten (vor der Eminentia) mit der Tibia zusammenhängt. Vorne erhebt es sich frei, so daß man mit einer feinen Sonde darunter gelangen kann. Es ist etwas beweglich. An der Oberfläche dieses Knochenstücks hängt der hintere Teil des Kreuzbandes. Ein Teil von Faserbündeln, die ungefähr dem mittleren Teile des Bandes entsprechen, ist spitzwinklig lateralwärts geknickt; die Knochenstückchen an ihren Enden sind dem hinteren Teile des Kreuzbandes angelagert. Vom medialen Meniskus ist ein $3 / 4 \mathrm{~cm}$ langes, zungenförmiges Fetzchen abgerissen, das frei über die beschriebene Grube ragt. An der Innenseite des medialen Condylus des Femur sieht man eine nicht ganz linsengroße Grube, deren Lage 


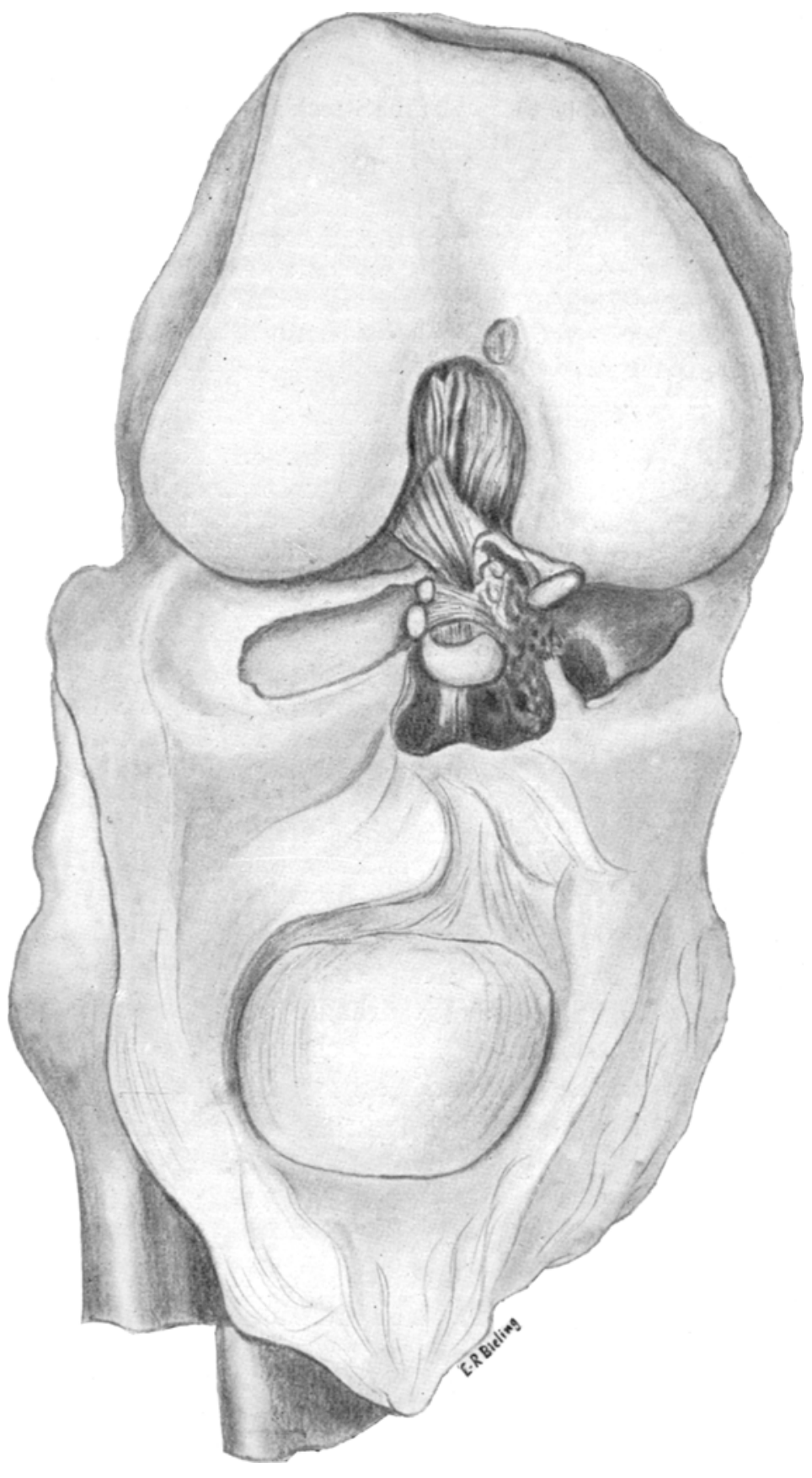

Fig. 2. 
am besten aus der Zeichnung hervorgeht. Macht man nun Bewegungen mit dem Gelenk, so legt sich beim Strecken das oben beschriebene, an dem gedrehten Stiel hängende Knochenstück in diese Grube hinein, beim Beugen verläßt es dieselbe.

Das zweite Präparat betrifft ebenfalls das rechte Kniegelenk eines Erwachsenen (Fig. 3). Das vordere Kreuzband, das sonst von regelrechter Beschaffenheit ist und ebenso wie der übrige Bandapparat keine Verletzung zeigt, hängt an einer ca. $2 \mathrm{~cm}$ breiten Knochenplatte, die es aus der Grube vor der Eminentia herausgerissen hat. Die Platte steht etwas schräg und sitzt hinten vor der Eminentia so fest an der Tibia, daß sie unbeweglich ist. Über sie hinweg ziehen Fasern vom Bande, in der Mitte vereinzelt nach den Seiten und hinten hin dichter werdend. Auffallend ist die Dicke der Platte; man hat den Eindruck, daß, wenn man sie in das Niveau, aus dem sie herausgerissen, wieder zurückdrücken wollte, sie nicht mehr hineinpassen würde. Der unterste Teil des Kreuzbandes, der sich an die Platte ansetzt, fühlt sich verhärtet an. Der durch die schräge Stellung des beschriebenen Knochenstücks bedingte Spalt beginnt am medialen Rande der äußeren Gelenkfläche der Tibia, setzt sich senkrecht zur Knochenachse medialwärts fort bis zum lateralen Rande der inneren Gelenkfläche der Tibia und biegt hier nach hinten um. Das Kreuzband hat aber auch ein zungenförmiges Stück aus dem lateralen Rande der medialen Gelenkfläche der Tibia abgerissen. An der Umbiegungsstelle des erwähnten Spaltes sieht man nämlich eine Knochenzacke hervorragen, von deren medialen Seite aus sich eine Fissur durch die mediale Gelenkfläche der Tibia nach hinten erstreckt bis zur Eminentia. Die Fissur verläuft anfangs parallel zur Ansatzlinie des Ligamentum cruc. ant., von der Mitte ab konvergiert sie mit ihr. Das zungenförmige Knochenstück ist unbeweglich.

An diese drei Beobachtungen knüpften sich sofort zwei interessante Fragen. Zunächst drängte sich mir die Frage auf: wie waren die Ausreißungen der Knochenstücke durch das vordere Kreuzband zustande gekommen?

Es ist ohne weiteres klar, daß eine der im Kniegelenk möglichen Bewegungen über das normale Maß hinausgetrieben worden war und dadurch die beschriebenen Verletzungen verursacht hatte. In Betracht kämen also die Hyperflexion, Hyperextension, Hyperpronation (gewaltsame Rotation nach innen), Hypersupination (gewaltsame Rotation nach außen), Hyperabduktion und Hyperadduktion. Durch die schon erwähnten über die Zerreißungen der Bänder im Kniegelenk angestellten Leichenexperimente ist -.. ich berücksichtige dabei besonders das vordere Kreuzband - folgendes festgestellt worden: 
Über die isolierte Ausreißung der Ligamenta cruciata des Kniegelenks. 23 I

I. H y perfle $\mathrm{x}$ j o n des Kniegelenks intakter Leichen, bis daß die Ferse das Gesäß berührt, läßt das vordere Kreuzband sowie

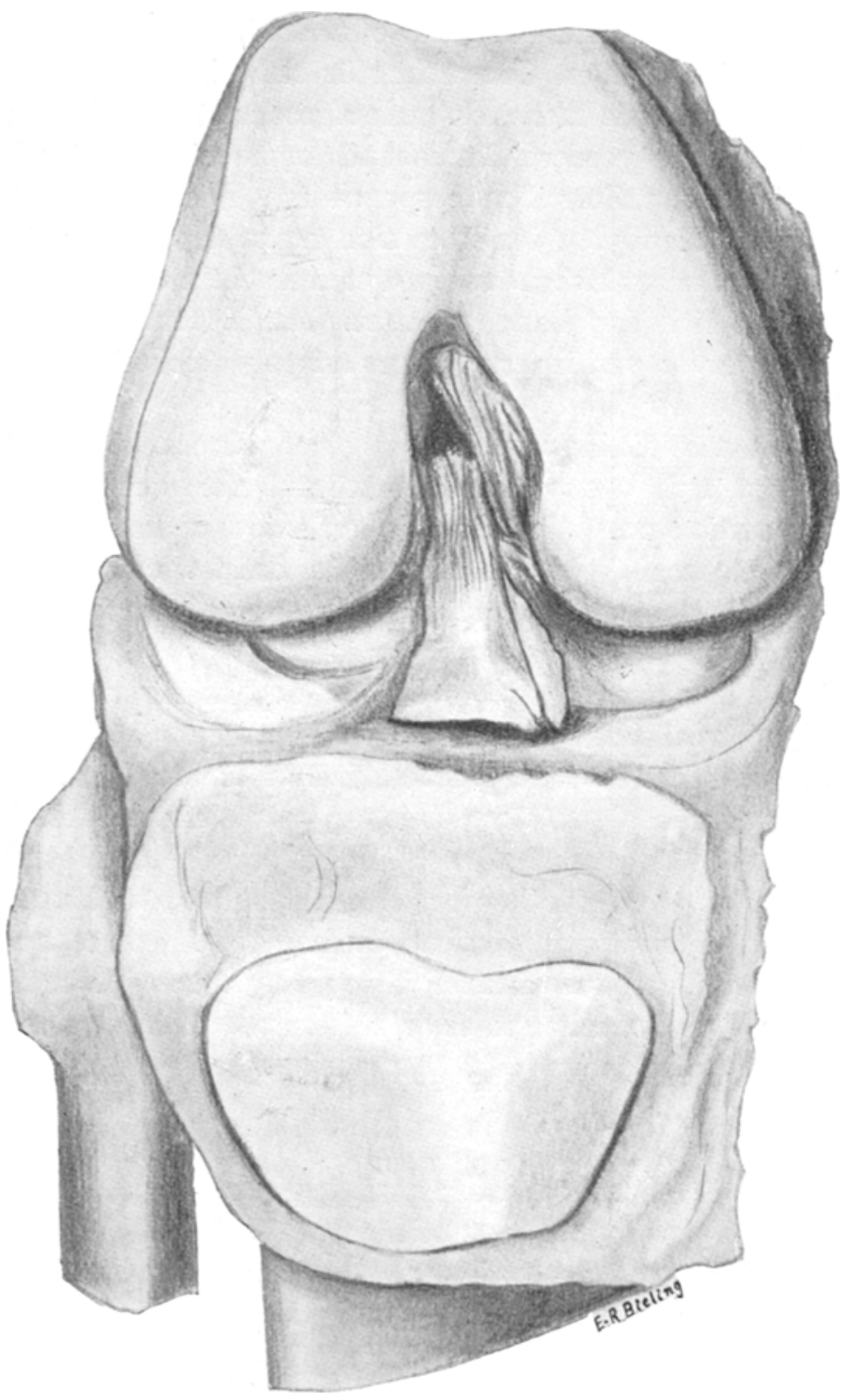

Fig. 3 . 
den gesamten Bandapparat vollkommen unversehrt. D i t t e 1 meint, da $B$ diese Bewegung bei gesunden und nicht gerade alten Menschen gar nicht als Hyperflexion zu betrachten sei, da ja die Orientalen die Sitte haben, nur so und nicht anders auf ihren Diwans zu sitzen. Den Grund hierfür sieht $\mathrm{P}$ a g e n s t e ch e r (1. c.) darin, daß, wie man sich am aufgeschnittenen Gelenk überzeugen kann, das vordere Kreuzband auch bei stärkster Hyperflexion nicht zum Zerreißen gespannt ist. Dies tritt erst dann ein, wenn die Kondylen der Tibia sich gegen den Femur stemmen und ein zweiarmiger Hebel aus der ersteren gebildet wird, an dessen kurzes Ende sich das Band anheftet. Am Lebenden oder unberührten Kadaver verhindern die massigen Weichteile des Oberschenkels und der Wade diese Hebelbildung.

Nimmt man die Hyperflexion an einem Knochenbandpräparat vor, so löst sich das vordere Kreuzband teilweise von seiner Femoralinsertion ab. Wird die Hyperflexion dadurch gesteigert, daß man einen Keil in die Kniekehle drückt und dann über diesen gewaltsam beugt, so löst sich das vordere Kreuzband v o 11 $\mathrm{s} t$ än dig von seiner Femoralinsertión $a b$.

Dieses Experiment mit dem Keil, das zuerst von Dit tel (1. c. S. 320) vorgenommen wurde, wird von $\mathrm{H}$ ö $\mathrm{n} \mathrm{i} \mathrm{g} \mathrm{s} \mathrm{c} \mathrm{h} \mathrm{m} \mathrm{i} \mathrm{e} \mathrm{d}$ (1. c. S. 589) falsch wiedergegeben, indem er berichtet, daß der Keil in die Kniekehle intakter Leichen gedrückt worden sei. Aus dem Wortlaut der betreffenden Stelle in der D i t t e l schen Abhandlung geht aber klar hervor, daß der Autor das Experiment am Knochenbandpräparat vorgenommen hat. Umso mehr möchte ich dies erwähnen, weil auch $\mathrm{P}$ a genste cher (l. c. S. 873) den Autor das Experiment an der intakten Leiche vornehmen läßt und dasselbe dann zum Ausgangspunkt eigener Versuche macht.

2. Hyperextension. Ein wesentlicher Unterschied, ob man die Versuche an intakten Leichen oder an präparierten Extremitäten vornimmt, besteht nicht. Wird der Unterschenkel nur wenig überstreckt, so reißt die hintere Kapselwand von der Umrandung der Tibia und das hintere Kreuzband vom Femur; das vordere Kreuzband und die Seitenbänder bleiben unversehrt. Wird die Uberstreckung gewaltsam ausgeführt, so zerreißt die hintere Kapselwand entsprechend den Kondylen des Oberschenkels 
und beide Kreuzbänder lösen sich ab, das vordere aus seiner Insertion an der Tibia, das hintere vom Femur. Dabei leistet jedes Kreuzband für sich allein der forzierten Streckung Widerstand. In den meisten Versuchen löst sich zuerst das vordere Kreuzband los und erst bei fortwirkender Gewalt das hintere. In einigen Fällen war es unmöglich, festzustellen, welches von den beiden Bändern sich zuerst losgelöst hatte. Nach $\mathrm{H}$ ön i g $\mathrm{s} \mathrm{ch} \mathrm{m}$ i e d (l. c.) sind die genannten Stellen, an denen die Lostrennung der Kreuzbänder erfolgt, konstant. Auffallend ist es nur, daß die von Dittel angestellten Versuche mit Hyperextension bezüglich der Kreuzbänder das gerade Gegenteil von den $\mathrm{H}$ ö $\mathrm{n}$ i g s chm i ed schen ergeben haben, nämlich Lostrennung des vorderen Kreuzbandes rom Femur und des hinteren von der Tibia.

3. H y p e r rot at i o n. Nach den obengenannten Autoren gelingt es sehr schwer, diese Bewegung im Kniegelenk übcr die physiologische Grenze hinaus auszuführen, da Ober- und Unterschenkel zu wenig Angriffspunkte bieten. Selbst wenn man den FuB als Winkelhebel mitbenützt, so ist er sehr bald aus seinem Talocruralgelenk ausgedreht. Dittel (1. c.) benutzte deshalb einen langen Keil, den er in den Zwischenknochenraum zwischen Schienbein und Wadenbein einstieB. D u breil und M a r tell i èr $\mathrm{e}^{1}$ ) bedienten sich bei jhren Versuchen eines eigens konstruierten Drehapparates, mit dessen Hilfe sie den Unterschenkel um die vertikale Achse nach aulien und innen rotierten.

a) Hyperpronation. An intakten Leichen gelingt es selten, durch Rotation nach innen eine Verletzung hervorzurufen. Am Knochenbandpräparat ruft diese Rotation folgende Verletzungen hervor. Wird sie n u $r$ w e n j g über die physiologische Grenze vorgenommen, so ist meistens die mediale, seltener die laterale Hälfte der vorderen Kapselwand an einer kleinen Stelle der Quere nach schlitzförmig eingerissen. Wird die Rotation s t ärker a us g e fü hr t, so erfolgt meistens eine Lostrennung der medialen Hälfte der vorderen und der lateralen Hälfte der hinteren Kapselwand, Losreißung des Lig. lateral int. (sowohl

I) Über dic Verrenkungen des Kniegelenks durch Rotation. Schmidts Jahrbücher 1853, Bd. 78 , S. 65. 
vom Femur als von der Tibia, ersteres anscheinend öfters), des Lig. lateral. extern vom Capitulum fibulae, des Lig. cruciat. ant. (sowohl vom Femur als von der Tibia); das Lig. cruciat. post. bleibt stets erhalten. Wird die Rotation mit großer Gewalt ausgeführt, so entsteht die ,Rotationsluxation des Unterschenkels nach innen", deren Symptome sowie die dabei entstandenen Verletzungen im Gelenk H ö n i g s c h m i e d (1. c. S. 6r6) eingehend schildert.

b) Hypersupination. Die Versuche an intakten Leichen und an präparierten Kniegelenken ergeben ziemlich ïbereinstimmende Resultate. Wird der Unterschenkel n u r we n i g über die physiologische Grenze nach außen rotiert, so erfolgt starke Anspannung und Ausdehnung der medialen Hälfte der vorderen Kapselwand. Gelegentlich zerreißen dabei die oberflächlichsten Bündel; seltener ist die Kapsel von Lig. lat. int. bis zum medialen Rande der Sehne des Extensor quadr. crur. von der Umrandung der Tibia losgetrennt. Von den Zwischenknorpeln ist meist bloß der innere von der Tibia losgerissen, der äußere bleibt stets an seinem Platze. Bei Rotation mit gro Be r Ge w a lt erfolgt meistens Losreißung des Lig. lat. int. von seiner Insertion am Femur, des Liq. cruc. ant. ebenfalls von seiner Femoralinsertion, und unvollkommene Lostrennung des Liq. cruc. post. von seiner Schienbeininsertion. Das Lig. lat. extern. bleibt stets erhalten. Wird die Supinationsbewegung noch weiter getrieben, so erhält man die "Rotationsluxation nach außen."

4. Hy perabduktion. Auch hier ergeben die Versuche an intakten Leichen dieselben Resultate wie am Knochenbandpräparat. Konstant reißt zunächst das Lig. lat. int. ab (meistens an der Insertion am Fcmur), dann das Lig. cruc. post. (dieses fast stets von seiner Femoralinsertion), hierauf das Lig. cruc. ant. (meistens von der Tibialinsertion), und mehr oder weniger die Kapsel. In den meisten Fällen kommt es auch zur Lostrennung des inneren, seltener des äußeren Zwischenknorpels. Das Lig. lat. extern. bleibt stets erhalten. Schließlich kommt es zum Losreißen des oberen Endes der Tibia. Fraktur des Oberschenkels oberhalb der Kondylen, Zermalmung des Condyl extern. tibiae et femoris, Lostrennung der Epiphyse vom unteren Ende des Femur, inkomplete Luxation des Unterschenkels nach außen. 
5. Hy peradduktion hat dieselbe Wirkung auf den Bandapparat wie die Hyperabduktion, nur daß die Verletzungen auf der entgegengesetzten Seite auftreten. Stets reißt zunächst das Lig. later. extern. (meistens vom Capit. fibulae), dann das Lig. cruc. ant. (meistens von der Insertion an der Tibia); hiernach das Lig. cruc. post. (meistens vom Femur), und je nach dem Grade der mitgeteilten Bewegung mehr oder weniger weit die Kapsel. Ziemlich häufig erfolgt Abhebung des äußeren, seltener beider Zwischenknorpel von der Tibia. Schließlich kommt es zu den bei der Hyperabduktion genannten analogen schweren Verletzungen.

Aus dem soeben Mitgeteilten ergibt sich zunächst, daß Hyperextension, Hyperrotation, Hyperabduktion und Hyperadduktion für die isolierte Ausreißung des Lig. cruc. ant. aus der Tibiainsertion nicht verantwortlich gemacht werden können, da sie stets mit anderen Verletzungen verbunden sind, während in unseren Präparaten — es sei dies nochmals ausdrücklich erwähnt sonst kcine Veränderung besteht. Die Hyperflexion ist die einzige Bewegung, die uns der Lösung der Frage näher bringt. Bei ihr sehen wir eine isolierte Ausreißung des vorderen Kreuzbandes zustande kommen, allerdings aus der Femoralinsertion. Es bleibt uns also nichts weiter übrig, als anzunehmen, da $B$ noch eine andere Gewalt eine wichtige Rolle beim Zustandekommen der in Frage stehenden Verletzung spielt. Diese Gewalt ist nun von Dit t e l (1. c.) bei einem Fall festgestellt und beschrieben worden. Ich will den Fall, auf den auch $\mathrm{P}$ a ge $\mathrm{n} \mathrm{s} t \mathrm{e} \mathrm{ch}$ e $\mathrm{r}$ in seiner Arbeit eingeht, kurz wiedergeben.

Ein Mann von 25 Jahren wird vom Wirt zur Türe hinausgeworfen in den Hof. Vor der Tür fällt er auf die Knie, so daß der linke Unterschenkel über den rechten zu liegen kam, sie kreuzten sich also hinten, während der Stamm auf die Kondylen des Oberschenkels sich stützte. In dieser Stellung trat ihn der Wirt in die linke Kniekehle. Es entstand ein starker Bluterguß in das Gelenk, der mehrfach punktiert wurde. Die Punktionen führten zu Sepsis, diese zur Amputation des Oberschenkels und etwas später zum Tode. Bei der Autopsie des Gelenkes wurde festgestellt, da $B$ das sonst regelrechte vordere Kreuzband vom Schienbein in der Weise abgelöst war, daß die ganze Eminentia intercondyl. mit einer ovalen Knochenscheibe, die $4 \mathrm{~cm}$ lang und $3 \mathrm{~cm}$ breit war, abgehoben war. Der gesamte übrige Bandapparat sowie die ganze innere Fläche des Gelenkes zeigten sich nur insofern erkrankt, 
als dies notwendigerweise aus der Entzündung und der Bespülung mit dem jauchigen Eiter resultierte.

Dieser Fall lehrt, daß eine isolierte Ausreißung des Lig. cruc. ant. aus seiner Tibiainsertion beim Lebenden dann zustandekommt, wenn bei sehr starker Hyperflexion eine Gewalt auf das Gelenkende des Schienbeins von rückwärts trifft und dadurch ein Weiterschieben des Schienbeins in der Richtung der Femoralachse verursacht. D i t t el konnte diese Erscheinung durch eine Reihe von Leichenexperimenten bestätigen. Durch die entgegengesetzte Gewaltwirkung konnte er — nebenbei bemerkt --- den Ausriß des hinteren Kreuzbandes mit daran hängendem Knochenstück von der Tibia hervorrufen.

Die Verletzungen an meinen beiden Präparaten sind von derselben Art wie die im Dittelschen Fall. Ich bin daher berechtigt, anzunehmen, da $B$ sie auch auf dieselbe soeben beschriebene Weise zustandegekommen sind.

Dies möchte ich jedoch nur mit einer gewissen Einschränkung sagen. Zunächst kann trotz des D i t t e l schen Falles und der sich an ihn anschließenden Leichenexperimente nicht bezweifelt werden, da $B$ das vordere Kreuzband beim Lebenden allein schon durch gewaltsame Überbeugung - also ohne Mitwirkung der den Unterschenkel von hinten nach vorn treibenden Kraft - isoliert zerreißen kann. Dies beweist einwandfrei die zweite von $\mathrm{P}$ a ge $\mathrm{n} \mathrm{s} \mathrm{t} \mathrm{e} \mathrm{ch} \mathrm{e} \mathrm{r} \mathrm{(l.} \mathrm{c.)} \mathrm{veröffentlichte} \mathrm{Krankengeschichte.}$

Es handelte sich um eine 56 jährige, sehr starke Dame, die etwa Io Stufen einer steilen Treppe in sitzender Stellung mit im Knie gebeugten und untergeschlagenen linken Bein hinuntergerutscht war. Sofort sehr heftige Schmerzen und Unmöglichkeit zu gehen. Am folgenden Tage geringer Hämarthros, bedeutende seitliche Beweglichkeit nach jeder Richtung und gesteigerte Rotation des Unterschenkels nach innen und außen, sowohl in Streckung als besonders in Beugung. Die Tibia läßt sich mit Leichtigkeit nach vorn luxieren. Vier Tage nach dem Unfall Eröffnung des Gelenks. In demselben wenig frisches und geronnenes Blut. Patella über den Condyl, med. fem. leicht zu luxieren. Das Lig. mucos. bis auf einen dünnen Streifen durchrissen, wird völlig getrennt. Auf dem Knorpel des Condyl. med. fem. sind einige, in der Richtung von vorn nach hinten verlaufende, etwa I cm lange Rißchen. Das Lig. cruc. ant. ist in der hinteren Partie seines Ansatzes am Condyl. lat. fem. durchtrennt, aber nicht ausgerissen. so daß ein kleiner Stumpf 
noch am Condylus haftet. Von der Rißstelle erstreckt sich ein Längsriß fast bis zur tibialen Insertion.

Dann aber habe ich mich beim Studium der Leichenexperimente nicht des Eindrucks erwehren können, daß die Experimente, so sorgfältig sie auch ausgeführt sein mögen, letzten Endes doch nicht so absolut sicher und einwandfrei sind und es auch nicht sein können.

Aus folgenden Gründen. Wir wissen, daß jedes Band, wenn es auch noch so stark ist, einen gewissen Grad von Dehnbarkeit und Nachgiebigkeit besitzt. Erst wenn die höchst zulässige Grenze derselben überschritten wird, wird eine Zerreißung bzw. Auslösung oder AusreiBung eintreten. Wo diese Grenze liegt, ist schwer oder gar nicht festzustellen, da sie von einer Reihe von Faktoren abhängig ist. Der Bandapparat ist bald schwächer, bald stärker entwickelt. Alter und Konstitution spielen hier eine wesentliche Rolle. Die Dehnung ist oft eine elastische, welche bei Aufhören des Zuges wieder zurückgeht und deshalb nachher nicht mehr nachweisbar ist. Auch die Beschaffenheit der Knochen ist zu berücksichtigen; bei den Knochenausreißungen ist es ein großer Unterschied, ob es sich um Knochen von alten oder jungen Individuen handelt. Von ganz hervorragender Bedeutung ist der Stärkegrad der zur Anwendung gebrachten Gewalt, den wir nur schwer messen können, so daß es sehr schwierig ist, sie zu regulieren und zu überwachen. Es wird jedesmal ein anderes Resultat erzielt werden, je nachdem die Gewalt in Form rascher Schläge oder Stöße, langsam dehnend oder ruckweise angreift. Dasselbe gilt, wenn sie nur einmal oder wiederholt einwirkt. Wir sind berechtigt zu sagen, daß unter gleichen Bedingungen auch immer dieselbe Verletzung erzeugt werden wird, sind aber nicht in der Lage, stets über die gleichen Bedingungen zu verfügen. Wir haben gar nicht die Mittel und die Macht, jeden einzelnen der oben genannten Faktoren, die auch für den Lebenden von derselben großen Bedeutung sind, von Fall zu Fall in gleicher Weise zur Geltung zu bringen, um absolut gleiche und sichere Resultate zu erzielen. Die wenn auch im ganzen übereinstimmenden, so doch öfters verschiedenen, manchmal sogar sich widersprechenden Ergebnisse in den Versuchsreihen - nicht nur des einzelnen Autors, sondern auch der Autoren untereinander - beweisen dies. Aus 
diesen Gründen möchte ich auch nicht mit voller Bestimmtheit behaupten, daß die Knochenausreißung in meinen Präparaten unter allen Umständen auf dieselbe Weise zustande gekommen sein muß wie im $\mathrm{D}$ it te lschen Fall.

Prüfen wir nach den soeben gemachten Ausführungen unter besonderer Berücksichtigung der Dehnungsmöglichkeit der Bänder in Kürze nochmals die sechs Bewegungsarten darauf hin, welche von ihnen die isolierte Ausreißung des vorderen Kreuzbandes aus der Tibiainsertion verursacht haben kann. so können wir zunächst die Hyperflexion ausscheiden, weil die Ausreißung hier stets am Femuransatz zustandekommt. Die Hyperextension hat wenig Wahrscheinlichkeit für sich, da die hintere Kapselwand schon nach geringer Überstreckung reißt. Das vordere Kreuzband löst sich hier aber erst nach gewaltsamer Uberstreckung. Selbst wenn wir also der Kapselwand einen hohen Dehnungsgrad zusprechen, würde dieser doch zweifellos bei der gewaltsamen Uberstreckung überschritten werden müssen und zur Zerreißung der Kapsel führen. Damit würde aber die der Erörterung zugrunde liegende Voraussetzung der Unversehrtheit des übrigen Bandapparates nicht mehr zutreffen. Dasselbe gilt von der Hyperpronation und Hypersupination. Bei diesen ist aber, ebenso wie bei der Hyperabduktion, außerdem noch zu berücksichtigen, daß wir das Vorausgehen der Dehnung von $z$ w e i Bändern annehmen müßten, beror es zum Abreißen des vorderen Kreuzbandes kommt. Ferner haben wir gesehen, da $B$ die Verletzungen meistens $\mathrm{n} \mathrm{a} \mathrm{ch}$ e i n a $\mathrm{n} d$ e r eintreten. Wenn also z. B. bei der Hyperabduktion die Gewalt zu wirken anfängt, so spannt sich zuerst das Lig. lat. int., dann das Lig. cruc. post. an. Bevor letzteres den höchsten Grad seiner Dehnungsfähigkeit erreicht hat, ist dies schon beim Seitenband geschehen und seine Zerreißung erfolgt; damit stimmt dann aber wieder nicht die Voraussetzung. - Dagegen können wir mit der Hyperadduktion das Zustandekommen der Verletzung erklären und ohne $Z$ wang von unsern Präparaten sagen, daß die unbekannte Gewalteinwirkung nach einer Dehnung des Lig. lat. extern. das Ausreißen des Lig. cruc. ant. verursacht hat; nachdem letzteres geschehen, hat sie aufgehört, so daß das Lig. cruc. post. verschont blieb; die Dehnung des Lig. lat. extern. war eine elastische, die nach Aufhören der Gewalt wieder zurückging und keine Spur hinterließ. 
Im Sinne einer Hyperadduktion hat meines Erachtens auch die Gewalt gewirkt, welche in dem von Geheimrat $\mathrm{Br}$ a u begutachteten Falle die Knochenausreißung aus dem Femur durch das vordere Kreuzband veranlaßte. Dem mit dem Anputzen der Stallwand beschäftigten Manne fiel ein Rind gegen die Innenseite des rechten Kniegelenks und bog dasselbe nach außen. Man kann annehmen, daß der Fuß oder der Unterschenkel sich in fixierter Stellung, eingeklemmt zwischen Rind und Wand, befand, als die Gewalt auf die Innenseite des Knies einwirkte. Außerdem hat der Mann vielleicht noch mit dem Oberschenkel eine Abwehrbewegung gemacht in einem der Gewalt entgegenwirkenden Sinne. Auch die Ausreißung aus der Femurinsertion würde der Erklärung durch Hyperadduktion nicht entgegenstehen, da, wie aus den Ausführungen sub 5 hervorgeht, die Ausreißung aus der Tibia allerdings meistens, aber nicht immer die Regel ist.

Fraglich erscheint mir nur, ob in diesem Falle die Ausreißung des vorderen Kreuzbandes die einzige Beschädigung des Bandapparates gewesen ist. Auf Grund der letzten Untersuchungsergebnisse halte ich nicht für ausgeschlossen, daß auch eine Verletzung des äußeren Seitenbandes vorgelegen hat.

Die zweite Frage, welche ich mir bei Betrachtung der beiden Präparate vorlegte, war die: haben diese seltenen Verletzungen schon $\mathrm{zu} \mathrm{L} \mathrm{e} \mathrm{b} \mathrm{z} \mathrm{e} \mathrm{it} \mathrm{e} \mathrm{n}$ bestanden oder sind sie erst an der Leiche zustande gekommen? Ich mußte also nach Merkmalen suchen, deren Vorkommen nur bei Lebenden möglich ist. In der Tat gelingt dies beiden Kniegelenken.

Zunächst lassen sich bei Präparat 2 sichere Zeichen eines Heilungsprozesses nachweisen, der nach der Verletzung eingesetzt hat. Schon bei der Beschreibung des Präparates hatte ich darauf aufmerksam gemacht, daß die ausgerissene Knochenplatte auffallend dick sei, und zwar so dick, daß sie in die Grube, aus der sie herausgerissen, nicht mehr hineinpassen würde, wenn man sie in dieselbe zurückdrücken wollte. Diese Verdickung ist meines Erachtens als kallöse Neubildung aufzufassen. Desgleichen spreche ich dic Vcrhärtung, welche man im untersten Teile des Kreuzbandes, welcher sich an die Knochenplatte ansetzt, findet, als Kallusbildung an. Schließlich ist die völlige Unbeweglichkeit der 
etwas schräg stehenden Platte aufzufassen als Folge einer festen Verwachsung, welche zwischen dem hinteren Teil ihrer Unterfläche und dem hinteren Teil der Grube, aus der sie gerissen, zustandegekommen ist.

Bei Präparat I sind Heilungsprozesse nicht nachweisbar, wohl aber Veränderungen, die nur durch den Gebrauch des Gelenks erklärbar sind. Dieselben finden sich an den herausgerissenen Knochenstückchen, welche, wie beschrieben, an den Fasern des Ligaments hängen.

Zunächst ist eine Formveränderung an ihnen festzustellen. Sie haben das Aussehen von herausgerissenen Knochenstücken verloren und eine mehr rundliche Form bekommen. Diese Form ist meines Erachtens durch ein Abschleifen zustande gekommen, dem die zwischen den Gelenkflächen befindlichen Knochenstückchen bei den Bewegungen des Kniegelenks ausgesetzt waren. Auch an ein gegenseitiges Abschleifen der Knochenstücke untereinander wäre $\mathrm{zu}$ denken.

Dann möchte ich auf die an der Innenseite des medialen Condyl. femor. befindliche linsengroße Grube aufmerksam machen, in welche sich das an dem gedrehten Stiel hängende größte Knochenstück bei Bewegungen beim Strecken hineinlegt und es beim Beugen wieder verläßt. Anzunehmen ist, daß dieses Knochenstück nach seiner Ausreißung sich bei jeder Streckbewegung an die Stelle der jetzigen Grube hinlegte, einen vorübergehenden, ganz leisen Eindruck machte und bei Beginn der Beugung dann wieder nach vorn glitt. Allmählich entstand auf diese Weise die Grube. Hiermit glaube ich bewiesen $z u$ haben, daß die Verletzungen bei beiden Kniegelenken schon $\mathrm{zu}$ L e bze it e n zustande gekommen waren.

Zum Schluß möchte ich noch darauf hinweisen, daß beide Gelenke uns zeigen, was aus den vom Ligament ausgerissenen und an ihm hängenden Knochenstückchen wird. I. Sie können fest anheilen wie im Präparat 2 und in der klinischen Beobachtung. 2. Wenn es sich um mehrere Knochenstücke handelt, können sie durch Abschleifen eine Formveränderung erfahren im Sinne einer Abrundung. 3. Wenn diese Formveränderung, wie ich ausgefürt habe, auf ein Abschleifen durch die Bewegungen im Gelenk bzw. gegeneinander zurückzuführen ist, so dürfte der Annahme nichts 
im Wege stehen, daß sie sich immer mehr abschleifen können, bis sie schließlich ganz verschwunden sind. Ich bin überzeugt, daß dies das Schicksal der winzigen Stückchen, die unter dem gestielten Knochenstück in Präparat I hängen, geworden wäre. 4. Sie lösen sich, wie wir aus Präparat I sehen können, von ihren Stielen allmählich $\mathrm{ab}$ und werden zu Gelenkkörpern, Gelenkmäusen. Die Erkenntnis dieser Art der Entstehung der traumatischen Gelenkkörper ist noch nicht alt. B a r th (l. c.) kommt das Verdienst zu, hierauf nachdrücklich hingewiesen zu haben. Schon bei der Beschreibung des Präparates habe ich auf das am meisten in das Gelenk hineinragende, erbsengroße Knochenstück, welches an einem ca. ${ }^{3 / 4} \mathrm{~cm}$ langen Stiel von Fasern hängt, aufmerksam gemacht und von diesem Stiel gesagt, daß er sich nach oben verschmälere und an seiner Abgangsstelle am Kreuzbande ganz dünn sei. Es unterliegt wohl keinem Zweifel, daß der Zeitpunkt nicht mehr fern war, an dem der Stiel durch die Bewegungen vollständig abgerissen und damit das Knochenstück zum Gelenkkörper geworden wäre. Ferner sahen wir, daß der Stiel um seine Achse gedreht ist. Dies zcigt uns, daß die Entstehung der traumatischen Gelenkkörper nicht nur durch Abreißung, sondern auch durch Abdrehung des Stiels zustande kommen kann. 